PROCEEDINGS OF THE

AMERICAN MATHEMATICAL SOCIETY

Volume 130, Number 9, Pages 2655-2660

S 0002-9939(02)06394-3

Article electronically published on February 4, 2002

\title{
A FEFFERMAN-POINCARÉ TYPE INEQUALITY FOR CARNOT-CARATHÉODORY VECTOR FIELDS
}

\author{
GIUSEPPE DI FAZIO AND PIETRO ZAMBONI
}

(Communicated by Christopher D. Sogge)

Dedicated to Professor Michele Frasca on the occasion of his sixtieth birthday

Abstract. In this note we prove a Fefferman-Poincaré type inequality in spaces with metric induced by Carnot-Carathéodory vector fields.

\section{INTRODUCTION}

In his celebrated paper, C. Fefferman [6] proved the inequality

$$
\int_{B}\left|u(x)-u_{B}\right|^{p}|V(x)| d x \leq c \int_{B}|\nabla u(x)|^{p} d x \quad \forall u \in C^{\infty}
$$

in the case $p=2$, assuming the potential $V$ to belong to the class $L^{r, n-2 r}, 1<r \leq$ $n / 2$. In (1.1) $u_{B}$ is the integral average $|B|^{-1} \int_{B} u(y) d y$, and $|B|$ is the Lebesgue measure of $B$.

Later, Chiarenza and Frasca [3] extended Fefferman's result, with a different proof, assuming $V$ in $L^{r, n-p r}, 1<r \leq n / p, 1<p<n$. Danielli, Garofalo and Nhieu in [5] introduced a suitable version of Morrey spaces adapted to the CarnotCarathéodory (C-C) metric and proved the same inequality with $V$ in the Morrey space $L^{1, \lambda}$ for some $\lambda>0$. Later, Danielli in [4] generalized [3] to the setting of $\mathrm{C}-\mathrm{C}$ metric.

A different approach to the inequality (1.1) was started with Schecter in [8], where he proved the inequality with $p=2$ and $V$ in the Stummel - Kato class. Subsequently, in [10], with a different and simpler proof, Schechter's result was extended assuming $V$ in a larger class containing the Stummel - Kato class. Later, in [11] inequality (1.1) was proved with $1<p<n$, and $V$ in a more general class of potentials generalizing [11] and giving applications to elliptic PDE. It is worthwhile to point out that the two approaches are essentially different one from each other and, except for some partial overlapping in the result, they are not comparable.

The aim of this note is to prove inequality (1.1) when the gradient in the righthand side is replaced by the energy associated to an arbitrary system of vector fields, and the function $V$ is taken in an appropriate Stummel-Kato class, defined using the Carnot-Carathéodory metric associated to the vector fields in a metric space.

Received by the editors July 21, 2000 and, in revised form, April 6, 2001.

2000 Mathematics Subject Classification. Primary 46E35; Secondary 35B60.

Key words and phrases. Fefferman-Poincaré inequality, Carnot-Carathéodory vector fields, Stummel-Kato class. 


\section{HyPOTHESES AND PRELIMINARY RESUlTS}

Let $(\mathcal{S}, \rho)$ be a metric space. A measure $\mu$ is called a doubling measure if there exists a constant $c_{D, \mu}>1$ such that

$$
\mu(B(x, 2 r)) \leq c_{D, \mu} \mu(B(x, r)), \quad x \in \mathcal{S}, r>0 .
$$

As usual we set $B(x, r)=\{y \in \mathcal{S}: \rho(x, y)<r\}$ to denote the metric ball of radius $r$ centered at $x$. When $B$ is a ball we shall write $x_{B}$ for the center and $r_{B}$ for the radius.

Let $X=\left(X_{1}, \ldots, X_{m}\right)$ a system of vector fields in $\mathcal{S}$. For a given function $u: \Omega \subset \mathcal{S} \rightarrow \mathbb{R}$, we set $X u=\left(X_{1} u, \ldots, X_{m} u\right)$ and $|X u|=\left(\sum_{j=1}^{m}\left(X_{j} u\right)^{2}\right)^{\frac{1}{2}}$, where, as usual, $X_{j} u(x)=\left\langle X_{j}, \nabla u(x)\right\rangle$, identifying the $X_{j}$ 's with the first order differential operator that acts on $u \in \operatorname{Lip}(\Omega)$ via the above formula.

Let $1 \leq p<\infty$. We define

$$
\mathcal{L}_{\mu}^{1, p}(\Omega)=\left\{u \in L_{\mu}^{p}(\Omega): X_{j} u \in L_{\mu}^{p}(\Omega), j=1, \ldots, m\right\} .
$$

For any $u \in \mathcal{L}_{\mu}^{1, p}(\Omega)$ we set

$$
\|u\|_{1, p, \mu}^{p}=\|u\|_{p, \mu}^{p}+\||X u|\|_{p, \mu}^{p} .
$$

As usual, the completion of the set $C_{0}^{\infty}(\Omega)$ with respect to the norm (2.1) will be denoted by $S_{0, \mu}^{1, p}(\Omega)$.

We now recall the definition of a Boman chain domain.

Definition 2.1. A domain $\Omega$ in $\mathcal{S}$ is said to satisfy the Boman chain condition of type $\sigma, M$, or to be a member of $\mathcal{F}(\sigma, M)$, if there exist constants $\sigma>1, M>1$, and a family $\mathcal{F}$ of metric balls $B \subset \Omega$ such that the following conditions are satisfied:

(i) $\Omega=\bigcup_{B \in \mathcal{F}} B$.

(ii) $\sum_{B \in \mathcal{F}} \chi_{\sigma B}(x) \leq M \chi_{\Omega}(x)$ for all $x \in \mathcal{S}$.

(iii) There is a so-called "central ball" $B_{0} \in \mathcal{F}$ such that for each ball $B \in \mathcal{F}$, there is a positive integer $k=k(B)$ and a chain of balls $\left\{B_{j}\right\}_{j=0}^{k}$ such that $B_{k}=B$ and each $B_{j} \cap B_{j+1}$ contains a ball $D_{j}$ for which $B_{j} \cup B_{j+1} \subset M D_{j}$.

(iv) $B \subset M B_{j}$, for all $j=0, \ldots, k(B)$.

Let $\mu$ and $\nu$ be two doubling measures in $(\mathcal{S}, \rho)$ with respect to metric balls, and let $\Omega$ be a domain in $\mathcal{S}$. We assume the following:

(H1) There is a constant $\alpha \geq 1$ such that for all balls $B$ with $\alpha B \subset \Omega$,

$$
\frac{1}{\nu(B)} \int_{B}\left|f-f_{B, \nu}\right| d \nu \leq c \frac{r_{B}}{\mu(B)} \int_{\alpha B}|X f| d \mu
$$

where the constant $c$ is independent of the function $f \in \mathcal{L}_{\mu}^{1, p}(\Omega)$. As usual $f_{B, \nu}$ denotes the average of the function $f$ with respect to the measure $\nu, f_{B, \nu}=$ $\nu(B)^{-1} \int_{B} f d \nu$, and the measures $\nu, \mu$ are related by the following balance condition: if $B$ and $\tilde{B}$ are balls with $\alpha B \subset \tilde{B} \subset \Omega$, then

$$
\frac{r_{B}}{r_{\tilde{B}}} \frac{\nu(B)}{\nu(\tilde{B})} \leq c \frac{\mu(B)}{\mu(\tilde{B})} .
$$

(H2) The measure $\mu$ on the right in (H1) satisfies a reverse doubling condition, i.e., there is a constant $c>0$ such that if $B_{1}$ and $B_{2}$ are balls with centers in $\Omega$ 
and with $B_{1} \subset B_{2}$, then

$$
\mu\left(B_{2}\right) \geq c \frac{r_{B_{2}}}{r_{B_{1}}} \mu\left(B_{1}\right) .
$$

(H3) The "segment" property holds for every ball $B \subset \Omega$, i.e., if $B$ is a subball of $\Omega$ with center $x_{B}$, then for each $x \in B$ there is a continuous curve $\gamma=\gamma_{x_{B} x}(t)$, $0 \leq t \leq 1$, in $B$ with $\gamma(0)=x_{B}, \gamma(1)=x$ and $\rho\left(x_{B}, z\right)=\rho\left(x_{B}, y\right)+\rho(y, z)$ for all $y, z \in \gamma$ with $y=\gamma(s), z=\gamma(t), 0 \leq s \leq t \leq 1$.

(H4) $\Omega$ is a Boman chain domain of type $\sigma, M$, i.e., $\Omega \in \mathcal{F}(\sigma, M)$.

In order to obtain our results we recall the following two theorems, proved in [7], that will play a crucial role in our proofs.

Theorem 2.2. Suppose that $\nu$ and $\mu$ are doubling measures on a metric space $(\mathcal{S}, \rho)$ and that the hypotheses (H1), (H2) and (H3) hold true for a ball $B_{0}$. Then for $\nu$-a.e. $x \in B_{0}$,

$$
\left|f(x)-f_{B_{0}, \nu}\right| \leq c \int_{B_{0}}|X f(y)| \frac{\rho(x, y)}{\mu(B(x, \rho(x, y)))} d \mu(y),
$$

where $c$ is a positive constant independent of $f, x$ and $B_{0}$.

The next theorem is a generalization of Theorem 2.2 to any Boman chain domain $\Omega$.

Theorem 2.3. Suppose that $\nu$ and $\mu$ are doubling measures on a metric space $(\mathcal{S}, \rho)$ and that hypotheses $\left(H_{1}\right)-\left(H_{4}\right)$ hold true for a domain $\Omega \subset \mathcal{S}$. Then for $\nu$-a.e. $x \in \Omega$,

$$
\left|f(x)-f_{B_{0}, \nu}\right| \leq C \int_{\Omega}|X f(y)| \frac{\rho(x, y)}{\mu(B(x, \rho(x, y)))} d \mu(y),
$$

where $B_{0}$ is the central ball in $\Omega$ and $C$ is independent of $f$ and $x$.

Definition 2.4 (Stummel-Kato class). Let $(\mathcal{S}, \rho)$ be a metric space. Let $\mu$ and $\nu$ be doubling measures with respect to metric balls.

Let $V: \mathcal{S} \rightarrow \mathbb{R}, r>0$. We set $\phi(r)$ equal to

$$
\sup _{x \in \mathcal{S}}\left(\int_{\rho(x, y)<r} \frac{\rho(x, y)}{\mu(B(x, \rho(x, y))}\left(\int_{\rho(z, x)<r}|V(z)| \frac{\rho(z, y)}{\mu(B(z, \rho(z, y))} d \nu(z)\right)^{\frac{1}{p-1}} d \mu(y)\right)^{p-1} .
$$

We say that a function $V: \mathcal{S} \rightarrow \mathbb{R}$ belongs to the space $\tilde{M}_{p}(\mathcal{S})$ if and only if $\phi(r)$ is finite for any $r>0$.

If, in addition, $\lim _{r \rightarrow 0+} \phi(r)=0$, then we say that $V$ belongs to the space $M_{p}(S)$.

Remark 2.5. The definition above gives back the classical Stummel-Kato class if we take $p=2, \mathcal{S}=\mathbb{R}^{n}, d \mu=d \nu=$ Lebesgue measure (e.g. [1], [2], [8] and [9]).

\section{MAin Results}

Theorem 3.1. Let $\nu$ and $\mu$ be two doubling measures on a metric space $(\mathcal{S}, \rho)$, and let hypotheses (H1), (H2) and (H3) hold true for a ball $B \subset \mathcal{S}$. Assume $V \in \tilde{M}_{p}(\mathcal{S})$. Then there exists a positive constant $c$, independent of $u$, such that

$$
\int_{B}|V(x)|\left|u(x)-u_{B, \nu}\right|^{p} d \nu(x) \leq c \phi\left(2 r_{B}\right) \int_{B}|X u(x)|^{p} d \mu(x),
$$

for any $u \in \mathcal{L}_{\mu}^{1, p}(B)$. 
Proof. By Theorem 2.2 and Fubini's Theorem we have

$$
\begin{gathered}
\int_{B}|V(x)|\left|u(x)-u_{B, \nu}\right|^{p} d \nu(x) \\
\leq c \int_{B}|V(x)|\left|u(x)-u_{B, \nu}\right|^{p-1}\left(\int_{B}|X u(y)| \frac{\rho(x, y)}{\mu(B(x, \rho(x, y))} d \mu(y)\right) d \nu(x) \\
\leq c \int_{B}|X u(y)|\left(\int_{B}|V(x)|\left|u(x)-u_{B, \nu}\right|^{p-1} \frac{\rho(x, y)}{\mu(B(x, \rho(x, y))} d \nu(x)\right) d \mu(y) \\
\leq c\left(\int_{B}|X u(y)|^{p} d \mu(y)\right)^{\frac{1}{p}} \\
{\left[\int_{B}\left(\int_{B}|V(x)|\left|u(x)-u_{B, \nu}\right|^{p-1} \frac{\rho(x, y)}{\mu(B(x, \rho(x, y))} d \nu(x)\right)^{\frac{p}{p-1}} d \mu(y)\right]^{\frac{p-1}{p}} .}
\end{gathered}
$$

We also have

$$
\begin{gathered}
\int_{B}\left(\int_{B}|V(x)|\left|u(x)-u_{B, \nu}\right|^{p-1} \frac{\rho(x, y)}{\mu(B(x, \rho(x, y))} d \nu(x)\right)^{\frac{p}{p-1}} d \mu(y) \\
\leq \int_{B}\left(\int_{B}|V(z)| \frac{\rho(z, y)}{\mu(B(z, \rho(z, y))} d \nu(z)\right)^{\frac{1}{p-1}} \\
\cdot\left(\int_{B}|V(x)|\left|u(x)-u_{B, \nu}\right|^{p} \frac{\rho(x, y)}{\mu(B(x, \rho(x, y))} d \nu(x)\right) d \mu(y) \\
\quad=\int_{B}|V(x)|\left|u(x)-u_{B, \nu}\right|^{p} \frac{\rho(x, y)}{\mu(B(x, \rho(x, y))} \\
\left(\int_{B}|V(z)| \frac{\rho(z, y)}{\mu(B(z, \rho(z, y))} d \nu(z)\right)^{\frac{1}{p-1}} d \mu(y) d \nu(x) \\
\leq \phi^{\frac{1}{p-1}}\left(2 r_{B}\right) \int_{B}|V(x)|\left|u(x)-u_{B, \nu}\right|^{p} d \nu(x) .
\end{gathered}
$$

From (3.1) and (3.2) we obtain the desired conclusion.

Remark 3.2. It is not difficult to extend the previous result to the case when $\Omega$ is a Boman chain domain of type $\sigma, M$. In fact, repeating the proof of Theorem 3.1 yields

Theorem 3.3. Let $\nu$ and $\mu$ be two doubling measures on a metric space $(\mathcal{S}, \rho)$, and let hypotheses (H1)-(H4) hold true for a domain $\Omega \subset \mathcal{S}$. Assume $V \in \tilde{M}_{p}(\mathcal{S})$. Then there exists a positive constant $c$, independent of $u$, such that

$$
\int_{\Omega}|V(x)|\left|u(x)-u_{B_{0}, \nu}\right|^{p} d \nu(x) \leq c \phi\left(2 M r_{B_{0}}\right) \int_{\Omega}|X u(x)|^{p} d \mu(x),
$$

where $B_{0}$ is the central ball in $\Omega$, for any $u \in \mathcal{L}_{\mu}^{1, p}(\Omega)$. 
However, we preferred to show the result in the case of metric balls in view of forthcoming applications to the regularity properties of the solutions to some PDEs.

The next corollaries are useful in connection with PDEs.

Corollary 3.4. Let the hypotheses of Theorem 3.1 hold true. Then there exists a positive constant $c$, independent of $u$, such that

$$
\int_{B}|V(x)||u(x)|^{p} d \nu(x) \leq c \phi\left(2 r_{B}\right) \int_{B}|X u(x)|^{p} d \mu(x), \quad \forall u \in S_{0, \mu}^{1, p}(B) .
$$

Corollary 3.5. Under the same assumptions of Theorem 3.1, for any $\varepsilon>0$ there exists a positive function $K(\varepsilon) \sim \frac{\varepsilon}{\left[\phi^{-1}(\varepsilon)\right]^{Q+p}}$ such that

$$
\int_{\Omega}|V(x)||u(x)|^{p} d \mu(x) \leq \varepsilon \int_{\Omega}|X u(x)|^{p} d \mu(x)+K(\varepsilon) \int_{\Omega}|u(x)|^{p} d \mu(x),
$$

for all $u \in S_{0, \mu}^{1, p}(\Omega)$, where $\Omega$ is a bounded subset of $\mathcal{S}$, containing the support of $V$, and $Q=\log _{2} c_{D, \mu}$.

Proof. Let $\varepsilon>0$. Let $r$ be a positive number that we will choose later. Let $\left\{\alpha_{i}^{p}\right\}$, $i=1,2, \ldots, N(r)$, be a finite partition of unity of $\bar{\Omega}$, such that $s p t \alpha_{i} \subseteq B\left(x_{i}, r\right)$, with $x_{i} \in \bar{\Omega}$. From Corollary 3.2 we have

$$
\begin{gathered}
\int_{\Omega}|V(x)||u(x)|^{p} d \nu(x)=\int_{\Omega}|V(x)||u(x)|^{p} \sum_{i=1}^{N(r)} \alpha_{i}^{p}(x) d \nu(x) \\
=\sum_{i=1}^{N(r)} \int_{\Omega}|V(x)||u(x)|^{p} \alpha_{i}^{p}(x) d \nu(x) \\
\leq \sum_{i=1}^{N(r)} c \phi(2 r)\left(\int_{\Omega}|X u(x)|^{p} \alpha_{i}^{p}(x) d \mu(x)+\int_{\Omega}\left|X \alpha_{i}(x)\right|^{p}|u(x)|^{p} d \mu(x)\right) \\
\leq c \phi(2 r)\left(\int_{\Omega}|X u(x)|^{p} d \mu(x)+\frac{N(r)}{r^{p}} \int_{\Omega}|u(x)|^{p} d \mu(x)\right) .
\end{gathered}
$$

Choosing $r$ such that $c \phi(2 r)=\varepsilon$ and observing that $N(r) \simeq r^{-Q}$, we get the corollary.

\section{ACKNOWLEDGEMENTS}

We wish to thank R. Wheeden for useful discussions and suggestions, and the anonymous referee, who helped us to improve the paper.

\section{REFERENCES}

[1] M. Aizenman and B. Simon, Brownian motion and Harnack inequality for Schrödinger operators, Comm. Pure Appl. Math. 35 (1982), 209-273. MR 84a:35062

[2] F. Chiarenza, E. Fabes and N. Garofalo, Harnack's inequality for Schrödinger operators and continuity of solutions, Proc. A.M.S. 98 (1986), 415-425. MR 88a:35037

[3] F. Chiarenza and M. Frasca, A remark on a paper by C. Fefferman, Proc. Amer. Math. Soc. 108 (1990), 407-409. MR 91a:46030

[4] D. Danielli, A Fefferman - Phong inequality and applications to quasilinear subelliptic equations, Potential Analysis 11 (1999), 387-413. MR 2000j:35044 
[5] D. Danielli, N.Garofalo and D.Nhieu, Trace inequalities for Carnot-Caratheodory spaces and applications, Ann. Scuola Norm. Sup. Pisa XXVII (1998), 195-252. MR 2000d:46039

[6] C. Fefferman, The uncertainty principle, Bull. Amer. Math. Soc. 9 (1983), 129-206. MR 85f:35001

[7] G. Lu and R. Wheeden, An optimal representation formula for Carnot-Carathéodory vector fields, Bull. London Math. Soc. 30 (1998), 578-584. MR 2000a:31005

[8] M. Schechter, Spectra of partial differential operators, North Holland (1986). MR 88h:35085

[9] C. Simader, An elementary proof of Harnack's inequality for Schrödinger operators and related topics, Math. Z 203 (1990), 129-152. MR 91m:35071]

[10] P. Zamboni, Some function spaces and elliptic partial differential equations, Le Matematiche 42 (1987), 171-178. MR 90k:46080

[11] P. Zamboni, Unique continuation for non negative solutions of quasilinear elliptic equations, Bull. of Australian Math. Soc. 64 (2001), 149-156. CMP 2001:16

Università di Catania, Dipartimento di Matematica, viale Andrea Doria 6, 95125 Catania, Italy

E-mail address: difazio@dmi.unict.it

Università di Catania, Dipartimento di Matematica, viale Andrea Doria 6,95125 Catania, Italy

E-mail address: zamboni@dmi.unict.it 